\title{
Surgical prevention of terminal neuroma and phantom limb pain: a literature review
}

\author{
Ronald N. Bogdasarian, Steven B. Cai, Bao Ngoc N. Tran, Ashley Ignatiuk, Edward S. Lee \\ Division of Plastic and Reconstructive Surgery, Department of Surgery, Rutgers New Jersey Medical School, Newark, NJ, USA
}

\begin{abstract}
The incidence of extremity amputation is estimated at about 200,000 cases annually. Over $25 \%$ of patients suffer from terminal neuroma or phantom limb pain (TNPLP), resulting in pain, inability to wear a prosthetic device, and lost work. Once TNPLP develops, there is no definitive cure. Therefore, there has been an emerging focus on TNPLP prevention. We examined the current literature on TNPLP prevention in patients undergoing extremity amputation. A literature review was performed using Ovid Medline, Cochrane Collaboration Library, and Google Scholar to identify all original studies that addressed surgical prophylaxis against TNPLP. The search was conducted using both Medical Subject Headings and free-text using the terms "phantom limb pain," "amputation neuroma," and "surgical prevention of amputation neuroma." Fifteen studies met the inclusion criteria, including six prospective trials, two comprehensive literature reviews, four retrospective chart reviews, and three case series/technique reviews. Five techniques were identified, and each was incorporated into a targetbased classification system. A small but growing body of literature exists regarding the surgical prevention of TNPLP. Targeted muscle reinnervation (TMR), a form of physiologic target reassignment, has the greatest momentum in the academic surgical community, with multiple recent prospective studies demonstrating superior prevention of TNPLP. Neurorrhaphy and transposition with implantation are supported by less robust evidence, but merit future study as alternatives to TMR.
\end{abstract}

Keywords Terminal neuroma / Phantom limb pain / Surgical prevention of phantom limb pain / Targeted muscle reinnervation / Targeted nerve implantation

Received: October 27, $2020 \bullet$ Revised: January 12, $2021 \bullet$ Accepted: February 8, 2021

pISSN: 2234-6163 • elSSN: 2234-6171 • https://doi.org/10.5999/aps.2020.02180 • Arch Plast Surg 2021;48:310-322
Correspondence: Edward S. Lee Division of Plastic Surgery, Rutgers New Jersey Medical School, 140 Bergen Street, Suite E1620, Newark, NJ 07103, USA

Tel: +1-973-972-5377

Fax: +1-973-972-8268

E-mail: leee9@njms.rutgers.edu

\section{INTRODUCTION}

The incidence of extremity amputation is estimated at 200,000 cases annually $[1,2]$. Of these, over $25 \%$ suffer from terminal neuroma or phantom limb pain (TNPLP), resulting in intractable pain, inability to wear a prosthetic device, and lost work $[3,4]$. Due to the significant disability generated by TNPLP, it is an area of growing scientific interest and investigation.

Despite a large body of literature on the management of estab- lished TNPLP, treatment outcomes remain unpredictable [428]. A convincing body of neuroscience literature supports the concept of cortical reorganization and the imprinting of peripheral pain onto the central pain centers [29-47]. This central maladaptation may explain the unpredictability of peripheral treatment of established TNPLP. The clinical and basic science data support a transition in approach from the treatment of established TNPLP to its prevention.

Both non-surgical and surgical preventative approaches have 
been explored. The results of pharmacological and physical therapy strategies for the prevention of TNPLP are unfortunately disappointing $[27,48-65]$. On the contrary, though small in number, TNPLP surgical prevention strategies have shown great success.

The purpose of this literature review is to evaluate the current body of literature on surgical techniques for the prevention of TNPLP in patients undergoing extremity amputation, and secondarily to propose a new target-based classification system for the surgical prevention of TNPLP.

\section{METHODS}

\section{Search strategy}

An online search was performed using Ovid Medline, Cochrane Collaboration Library, and Google Scholar to identify all original articles that addressed surgical prophylaxis against TNPLP. The following search terms were used: "phantom limb pain," "amputation neuroma," and "surgical prevention of amputation neuroma." The search was limited to articles in English from inception to 2019. Studies regarding non-surgical strategies, animal studies, or surgical strategies for the treatment of established neuroma or phantom limb pain were excluded. After removal of all duplications, citations from all three search engines were subjected to title and abstract screening by two independent reviewers (RNB and SBC). The full-text articles of studies that met the inclusion criteria were subsequently obtained and reviewed independently by two reviewers. Disagreement in data collection was resolved by consensus with a third evaluator (BNNT).

\section{Data collection}

The final list of studies was independently evaluated by two independent reviewers (RNB and SBC) and the following variables were extracted: article identifiers, technique category, purpose, design, sample size, analysis techniques, quantitative results, strengths, and limitations. The original two researchers independently reviewed each article and compiled their own tables. These were then combined and submitted to the principal investigator for final review.

\section{Classification of techniques}

A recent literature review introduced a classification system for surgical prophylaxis against TNPLP, according to which techniques are divided into the categories of end closure, transposition with implantation, neurorrhaphy, and alternate target reinnervation [11]. We proposed the addition of a new category, preservation of continuity, and subsequently re-categorized these approaches based on the target condition to ensure con- sistent terminology throughout the review. We opted to rename "alternate target reinnervation" as "targeted muscle reinnervation" due to the increasing prevalence of the latter term in the literature. The proposed target-based classification system, which is shown in Fig. 1 and demonstrated in Fig. 2, includes the target deficient, target reassignment, and target in continuity techniques.

\section{Target deficient}

In this category, the severed nerve is not coapted to another nerve. Rather, the severed nerve ending is closed off or buried. Transposition with implantation is a common strategy in which terminal nerves are buried into muscle, bone, or fascia so that their nerve endings grow into stable, deep structures rather than exposed pain receptors in the skin [11]. The end closure technique refers to manipulation of the epineurium to enclose the nerve's fascicles.

\section{Target reassignment}

In target reassignment, a severed nerve is coapted to a new nerve to provide natural ingrowth within a nerve sheath. This can be physiologic or non-physiologic, as described below.

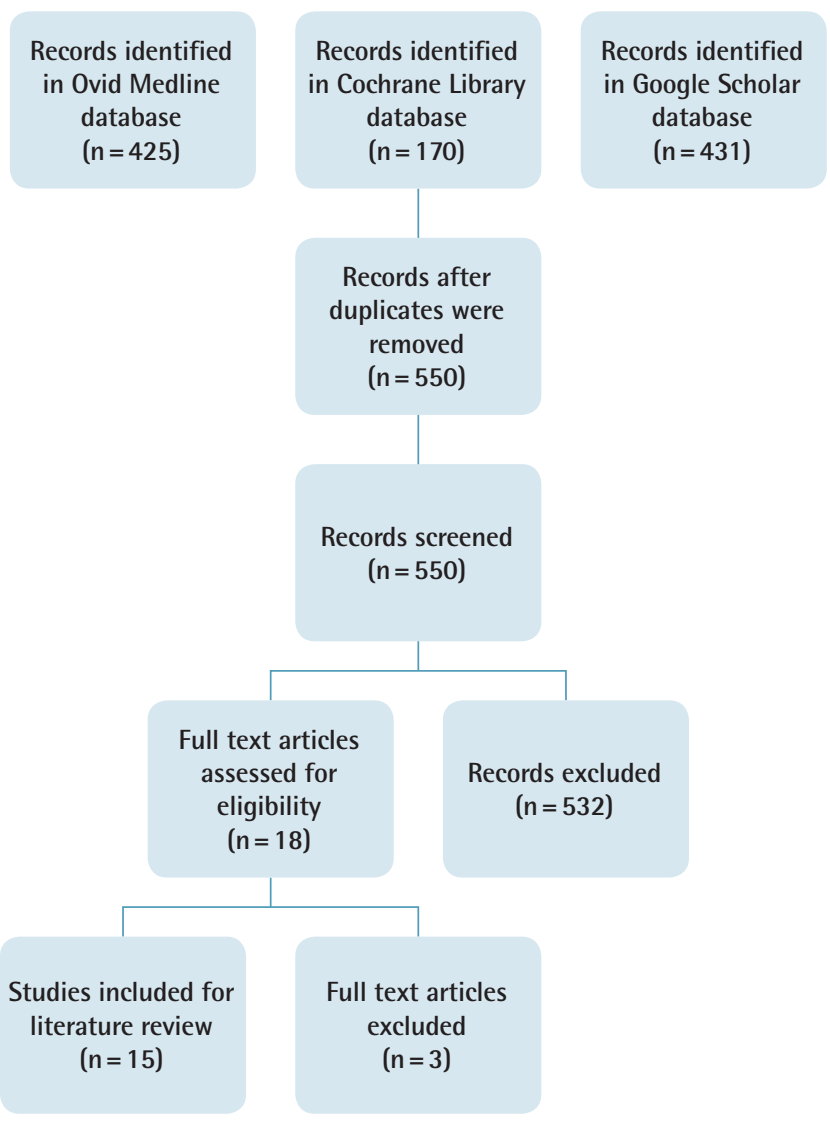

Fig. 1. Flow diagram. 


\section{Target Deficient}

End Closure: Epineurium used to "cap" terminal nerve

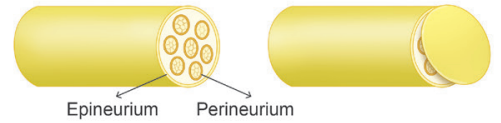

Transposition with Implantation
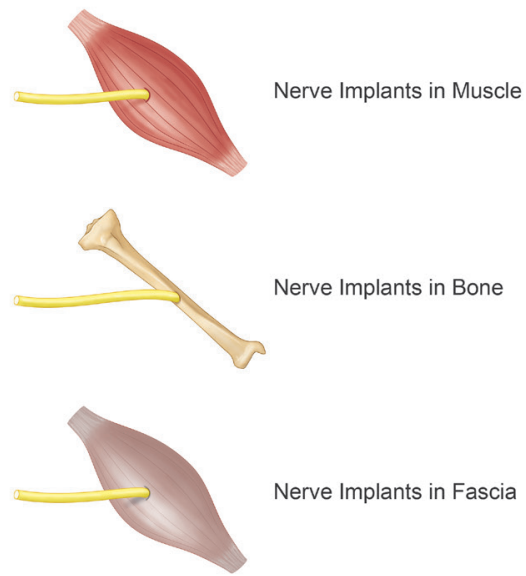

Nerve Implants in Fascia

\section{Target in Continuity}

Presentation of Continuity

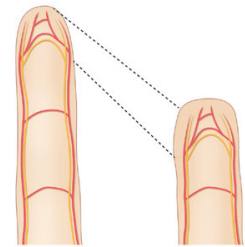

\section{Target Reassignment}

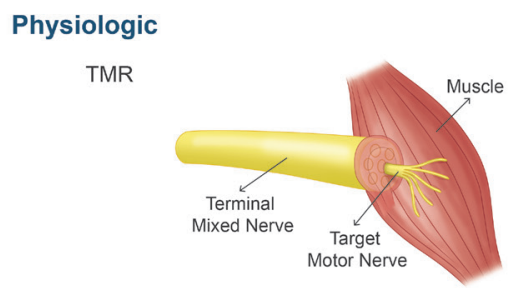

RPNI

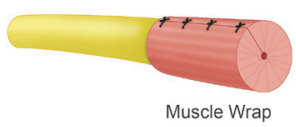

TNI

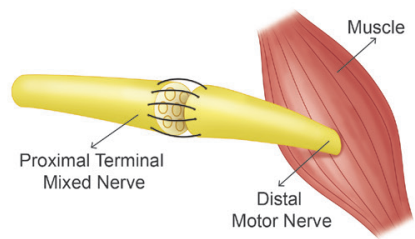

Non-Physiologic

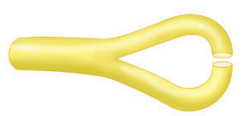

Single nerve is split \& coapted to itself

Rotationplasty

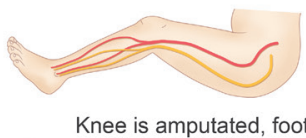

Knee is amputated, foot \& ankle are rotated $180^{\circ}$ $\&$ reattached proximally. Neurovascular bundle is left intact.

Fig. 2. Schematic of techniques. TMR, targeted muscle reinnervation; RPNI, regenerative peripheral nerve interface; TNI, targeted nerve implantation.

In physiologic target reassignment, the proximal severed nerve is coapted to a distal nerve attached to an end organ with its normal peripheral nerve endings. This theoretically provides the severed nerve with a normal end organ to grow into, reducing the changes of TNPLP.

The most popular version of this technique is known as targeted muscle reinnervation (TMR). TMR is the coaptation of proximal, terminal mixed nerves to distal, end motor nerves, providing a distal target for regenerating terminal axons. Notably, the distal, end motor nerve, once transected, intrinsically leads into a denervated segment of muscle, which is posited to attract physiologic nerve ingrowth into the muscle. TMR was developed for the purpose of creating functional electromyogram signals for integrated, controllable prosthetic devices $[6,28]$. Similarly, targeted nerve implantation (TNI) is the coaptation of proximal, terminal mixed nerves into distal motor nerves solely for the purpose of neuroma prevention [15].

An exception to the above definition is the regenerative peripheral nerve interface (RPNI) technique. In RPNI, a severed nerve is implanted directly into a non-vascularized muscle graft. This denervated muscle graft provides the severed nerve with an end organ to grow into in a physiologically natural way $[21,66]$. This theoretically reduces the risk of TNPLP. Like TMR, this reinnervation has been demonstrated by electromyogram signals.

In non-physiologic target reassignment, two proximal severed nerves are coapted, or a single nerve is split and coapted to itself. While these techniques provide a nerve sheath for axon ingrowth, they do not lead to a physiologic, distal end organ. These techniques are termed neurorrhaphy. Centro-central union (CCU) is an analogous term commonly found in the literature $[5,67]$. 


\section{Target in continuity}

These techniques can only be utilized when intermediate extremity components are amputated, while the proximal and distal tissues are preserved. Target in continuity techniques preserve the continuity of nerves and blood vessels to the terminal end organs, so that the amputation defect is reconstructed by the intact, distal parts. This category includes preservation of continuity, rotationplasty, and "spare parts" free tissue transfer.

Preservation of continuity is most frequently described for interphalangeal joint amputations of the digits. In this technique, the distal pulp, connected to the neurovascular bundles, is transposed proximally and sewn to the proximal amputation stump after removal of the intervening tissues. Thus, the wound is reconstructed with robust, vascularized, and innervated tissue. In this technique, the proper digital nerves are never severed; theoretically, there is no opportunity for neuroma formation $[68,69]$.

Rotationplasty of the lower limb is a procedure in which the knee is amputated, and the foot and ankle are rotated $180^{\circ}$ and reattached proximally. The tibia is fixated to the femur so that the reversed ankle joint acts as a pseudo-knee, allowing the transfer of power through a specialized prosthetic device.

Another strategy is the use of "spare parts," or a fillet flap to cover an amputated part. This technique may be used in the setting of trauma, where only some of the distal tissues are viable and useful for reconstruction - usually to preserve the length of the traumatized extremity. The distal tissues are dissected, maintaining their connections to arteries and veins. This fillet flap is transferred to the wound and the blood vessels are then anastomosed. The nerves can be coapted surgically to prevent future neuropathic wounds and, theoretically, to prevent TNPLP.

\section{RESULTS}

The Ovid Medline search returned 425 PubMed-indexed articles, from which only 10 articles met the inclusion criteria. The Cochrane Library database search produced 170 articles. No Cochrane Reviews on the topic exist and none of the articles met the inclusion criteria. The Google Scholar search produced 431 articles, from which one article met the inclusion criteria. There were four other articles that were found by reference searching. A total of 15 articles met the inclusion criteria and are summarized in Table 1.

There was one study on the target deficient technique (end closure, $n=1$ ), 12 studies on target reassignment (physiologic TMR, $n=8$; RPNI, $n=1$; TNI, $n=0$; non-physiologic neurorrhaphy/CCU, $n=3$ ), and two studies on target continuity (preservation of continuity, $\mathrm{n}=2$; rotationplasty, $\mathrm{n}=0$; fillet flap, $\mathrm{n}=0$ ). There were six prospective trials, two comprehen- sive literature reviews, four retrospective chart reviews, and three case series/technique reviews.

\section{Target deficient}

This literature review identified no studies evaluating the use of transposition with implantation in the prophylactic setting. A single article by Yuksel et al. [70] testing the end closure technique met the inclusion criteria. Yuksel et al. performed a prospective, randomized clinical trial including 23 patients and 48 nerves to compare epineural ligatures, flaps, and grafts for the prevention of amputation stump neuroma. The Tinel sign was used to elicit pain, which was recorded on a subjective 10-point visual analog scale for pain. The grafts, applied by excising a piece of epineurium and suturing it over the terminal nerve, demonstrated the greatest reduction in pain, with a pain score of 2.06 compared for 5.18 for ligatures and 4.25 for flaps $(\mathrm{P}<0.05)$. In summary, this study utilized the residual epineurium in three different ways and subjectively compared the prevention of neuroma formation. While grafts performed best, none of the techniques demonstrated excellent control [70]. This study had several limitations, including heterogeneous amputation causes, no clear statement of whether the study was blinded, the use of a subjective pain scale, and no histological confirmation of results.

\section{Target reassignment}

\section{Non-physiologic target reassignment}

This review identified three studies evaluating neurorrhaphy or $\mathrm{CCU}$ in the preventative setting. A nonrandomized, nonblinded, noncomparative prospective cohort study by Gorkisch et al. [9] in 1984 examined the efficacy of CCU for the prevention of hand neuroma. This group reported strong, albeit subjective results of this technique, with only one of 30 patients returning with clinical neuroma over a 4-year follow-up period.

Belcher and Pandya (2002) [67] performed a prospective, randomized, double-blinded clinical trial comparing CCU to simple transection in digital nerves. While half of the patients were lost to follow-up, objective tenderness measures demonstrated significant superiority of CCU $(\mathrm{P}<0.02)$.

Economides et al. (2016) [71] performed a small, prospective pilot study comparing tibial and common peroneal neurorrhaphy with collagen wrapping to nerve transection. This study demonstrated the superiority of $\mathrm{CCU}$ in multiple subjective and objective outcomes including the Tinel sign $(\mathrm{P}=0.03)$ and ambulation with a prosthetic device $(\mathrm{P}=0.01)$.

\section{Physiologic target reassignment}

There were nine articles on physiologic target reassignment techniques, with TMR being the most prominent area of study 
Table 1. Summary of articles meeting the inclusion criteria on the surgical prevention of terminal neuroma and phantom limb pain

\begin{tabular}{|c|c|c|c|c|c|c|c|c|}
\hline Author & $\begin{array}{l}\text { Category/ } \\
\text { Technique }\end{array}$ & Purpose & Design & Sample size & $\begin{array}{l}\text { Analysis } \\
\text { techniques }\end{array}$ & Quantitative results & Strengths & Limitations \\
\hline $\begin{array}{l}\text { Yuksel } 1997 \\
\text { [70] }\end{array}$ & $\begin{array}{l}\text { Target deficient/ } \\
\text { End closure }\end{array}$ & $\begin{array}{l}\text { To compare } \\
\text { epineural } \\
\text { ligatures, flaps } \\
\text { and grafts for } \\
\text { the prevention of } \\
\text { amputation } \\
\text { stump neuromas }\end{array}$ & $\begin{array}{l}\text { Prospective, } \\
\text { randomized } \\
\text { clinical trial } \\
\end{array}$ & $\begin{array}{r}23 \text { Patients, } \\
48 \text { nerves }\end{array}$ & $\begin{array}{l}\text { Tinel's sign used to } \\
\text { elicit pain, } \\
\text { recorded on a } \\
\text { subjective 10-point } \\
\text { VAS for pain. } \\
\text { ANOVA }\end{array}$ & $\begin{array}{l}\text { Pain scores 0-10: } \\
\text { Ligatures } 5.18 \\
\text { Flaps } 4.25 \\
\text { Grafts } 2.06 \\
\text { Grafts resulted in } \\
\text { significantly less } \\
\text { neuroma pain } \\
P<0.05\end{array}$ & $\begin{array}{l}\text { Single surgeon, } \\
\text { randomized, } \\
\text { varied } \\
\text { techniques in } \\
\text { single patients. } \\
\text { >6 Months } \\
\text { follow up }\end{array}$ & $\begin{array}{l}\text { Heterogeneous } \\
\text { amputation causes, } \\
\text { unclear if blinded, } \\
\text { subjective pain } \\
\text { scale }\end{array}$ \\
\hline $\begin{array}{l}\text { Gorkisch } \\
1984 \text { [9] }\end{array}$ & $\begin{array}{l}\text { Target } \\
\text { reassignment: } \\
\text { non- } \\
\text { physiologic/ } \\
\text { Neurorrhaphy }\end{array}$ & $\begin{array}{l}\text { To examine the } \\
\text { efficacy of } \\
\text { centro-central } \\
\text { nerve union for } \\
\text { the prevention of } \\
\text { hand neuromas }\end{array}$ & $\begin{array}{l}\text { Prospective, } \\
\text { nonrandomized, } \\
\text { nonblinded } \\
\text { cohort }\end{array}$ & 30 Patients & $\begin{array}{l}\text { Physical } \\
\text { examination, } \\
\text { subjective reports }\end{array}$ & $\begin{array}{l}\text { Only } 1 \text { of } 30 \text { patients } \\
\text { returned with } \\
\text { clinical neuroma } \\
\text { over } 4 \text { years follow } \\
\text { up. }\end{array}$ & Prospective & $\begin{array}{l}\text { No statistical analysis } \\
\text { or raw data } \\
\text { presented. Selection } \\
\text { bias, } \\
\text { nonrandomized, } \\
\text { nonblinded, } \\
\text { assessor bias }\end{array}$ \\
\hline $\begin{array}{l}\text { Belcher } \\
2000 \text { [67] }\end{array}$ & $\begin{array}{l}\text { Target } \\
\text { reassignment: } \\
\text { non- } \\
\text { physiologic/ } \\
\text { Neurorrhaphy }\end{array}$ & $\begin{array}{l}\text { To compare direct } \\
\text { digital nerve CCU } \\
\text { to simple nerve } \\
\text { transection } \\
\text { (control) in finger } \\
\text { amputations }\end{array}$ & $\begin{array}{l}\text { Prospective } \\
\text { randomized } \\
\text { double-blinded } \\
\text { clinical trial }\end{array}$ & $\begin{array}{l}31 \text { Digits } \\
\text { (control: 16, } \\
\text { CCU: } 15)\end{array}$ & $\begin{array}{l}\text { Subjective } \\
\text { questionnaire. } \\
\text { Objective S2PD, } \\
\text { Dolorimeter, grip } \\
\text { strength. } \\
\text { Students t-test, } \\
\text { Mann-Whitney test } \\
\end{array}$ & $\begin{array}{l}\text { Subjective sensation } \\
\text { better in control } \\
\text { than } \mathrm{CCU} \\
(\mathrm{P}<0.02) \text {. } \\
\text { Objective } \\
\text { tenderness better } \\
\text { in } \mathrm{CCU} \text { than control } \\
(\mathrm{P}<0.001) \text {. Grip } \\
\text { strength equal. }\end{array}$ & $\begin{array}{l}\text { Prospective, } \\
\text { randomized, } \\
\text { objective } \\
\text { measures, } \\
\text { double-blinded. } \\
2 \text { Years follow up }\end{array}$ & $\begin{array}{l}\text { Transfer bias (half lost } \\
\text { to follow up) } \\
\end{array}$ \\
\hline $\begin{array}{r}\text { Economides } \\
2016 \text { [71] }\end{array}$ & $\begin{array}{l}\text { Target } \\
\text { reassignment: } \\
\text { non- } \\
\text { physiologic/ } \\
\text { Neurorrhaphy }\end{array}$ & $\begin{array}{l}\text { To propose tibial } \\
\text { and common } \\
\text { peroneal nerve } \\
\text { coaptation at the } \\
\text { time of } \\
\text { amputation as } \\
\text { means to prevent } \\
\text { TNPLP }\end{array}$ & $\begin{array}{l}\text { Prospective } \\
\text { cohort } \\
\end{array}$ & $\begin{array}{l}\text { Coaptation } \\
\text { cohort: } 6 \\
\text { Traction } \\
\text { neurectomy } \\
\text { control: } 11\end{array}$ & $\begin{array}{l}\text { VAS, neuropathic } \\
\text { pain medication } \\
\text { use, neuroma } \\
\text { formation, } \\
\text { presence of } \\
\text { phantom limb pain, } \\
\text { prosthetic } \\
\text { tolerance, and } \\
\text { ambulatory status; } \\
\text { 2-tailed, unpaired } \\
\text { t-test and chi } \\
\text { square test for } \\
\text { data containing } \\
\text { continuous and } \\
\text { categorical } \\
\text { variables }\end{array}$ & $\begin{array}{l}6 \text { Months: VAS } \\
\text { scores (0.75 vs. } \\
5.6 ; \mathrm{P}=0.02) \text { as } \\
\text { well as neuroma } \\
(0 \% \text { vs. } 54.5 \% ; \\
\mathrm{P}=0.03) \text { and } \\
\text { phantom pain ( } 0 \% \\
\text { vs. } 63.6 \% ; \\
\mathrm{P}=0.01) \text { remained } \\
\text { lower among } \\
\text { patients who } \\
\text { underwent } \\
\text { coaptation. At } \\
\text { follow-up, } 67 \% \text { of } \\
\text { coaptation patients } \\
\text { were ambulating } \\
\text { with a prosthesis } \\
\text { vs. } 9 \% \text { of } \\
\text { neurectomy } \\
\text { patients ( } \mathrm{P}=0.01)\end{array}$ & Prospective & $\begin{array}{l}\text { No disclosure of } \\
\text { randomization, } \\
\text { blindness, patient } \\
\text { demographics } \\
\text { comparison or } \\
\text { number of surgeons } \\
\text { involved }\end{array}$ \\
\hline $\begin{array}{c}\text { Pet } 2014 \\
\text { [15] }\end{array}$ & $\begin{array}{l}\text { Target } \\
\text { reassignment: } \\
\text { physiologic/ } \\
\text { Targeted nerve } \\
\text { implantation }\end{array}$ & $\begin{array}{l}\text { To investigate if } \\
\text { TNI prevents } \\
\text { neuroma } \\
\text { formation in } \\
\text { acute traumatic } \\
\text { amputation }\end{array}$ & $\begin{array}{l}\text { Retrospective } \\
\text { chart review }\end{array}$ & 12 Patients & $\begin{array}{l}\text { Chart review for } \\
\text { palpable neuroma } \\
\text { pain }\end{array}$ & $\begin{array}{l}11 \text { of } 12 \text { patients } \\
\text { were free of } \\
\text { palpable neuroma } \\
\text { pain }\end{array}$ & $\begin{array}{l}>8 \text { Months follow } \\
\text { up }\end{array}$ & $\begin{array}{l}\text { Retrospective, } \\
\text { nonrandomized, } \\
\text { subjective pain } \\
\text { data, transfer bias, } \\
\text { selection bias, } \\
\text { assessor bias }\end{array}$ \\
\hline $\begin{array}{l}\text { Souza } 2014 \\
\text { [19] }\end{array}$ & $\begin{array}{l}\text { Target } \\
\text { reassignment: } \\
\text { physiologic/ } \\
\text { Targeted muscle } \\
\text { reinnervation }\end{array}$ & $\begin{array}{l}\text { To evaluate the } \\
\text { effect of TMR on } \\
\text { residual } \\
\text { neuroma pain }\end{array}$ & $\begin{array}{l}\text { Retrospective } \\
\text { chart review }\end{array}$ & $\begin{array}{l}11 \text { Without } \\
\text { neuroma; } \\
15 \text { with } \\
\text { established } \\
\text { neuroma }\end{array}$ & $\begin{array}{l}\text { Chart review for } \\
\text { complete } \\
\text { resolution of pain } \\
\text { and fit with TMR- } \\
\text { controlled } \\
\text { prosthesis }\end{array}$ & $\begin{array}{l}\text { None of the } 11 \\
\text { patients who } \\
\text { presented without } \\
\text { neuroma } \\
\text { developed a } \\
\text { neuroma after TMR }\end{array}$ & $\begin{array}{l}>6 \text { Months follow } \\
\text { up }\end{array}$ & $\begin{array}{l}\text { Retrospective. } \\
\text { Neuroma prevention } \\
\text { not a primary } \\
\text { objective of the } \\
\text { study. TMR primarily } \\
\text { performed for } \\
\text { control of upper } \\
\text { extremity } \\
\text { prosthetics, not } \\
\text { neuroma } \\
\text { prevention. No } \\
\text { objective pain scale }\end{array}$ \\
\hline
\end{tabular}


Table 1. Continued

\begin{tabular}{|c|c|c|c|c|c|c|c|c|}
\hline Author & $\begin{array}{l}\text { Category/ } \\
\text { Technique }\end{array}$ & Purpose & Design & Sample size & $\begin{array}{l}\text { Analysis } \\
\text { techniques }\end{array}$ & Quantitative results & Strengths & Limitations \\
\hline $\begin{array}{l}\text { Bowen } 2017 \\
\text { [6] }\end{array}$ & $\begin{array}{l}\text { Target } \\
\text { reassignment: } \\
\text { physiologic/ } \\
\text { Targeted muscle } \\
\text { reinnervation }\end{array}$ & $\begin{array}{l}\text { To show that TMR, } \\
\text { alone and in } \\
\text { conjunction with } \\
\text { other methods, } \\
\text { is a reliable } \\
\text { treatment for } \\
\text { terminal } \\
\text { neuroma and } \\
\text { phantom limb } \\
\text { pain }\end{array}$ & $\begin{array}{l}\text { Literature review } \\
\text { and } \\
\text { presentation of } \\
\text { experience }\end{array}$ & 20 Patients & Not disclosed & Not disclosed & $\begin{array}{l}\text { Well organized, } \\
\text { thorough }\end{array}$ & $\begin{array}{l}\text { No disclosure of } \\
\text { article selection } \\
\text { protocol in primary } \\
\text { publication, } \\
\text { prevention of } \\
\text { neuromas or } \\
\text { phantom limb pain } \\
\text { was not purpose of } \\
\text { review }\end{array}$ \\
\hline $\begin{array}{c}\text { Ives } 2017 \\
{[11]}\end{array}$ & $\begin{array}{l}\text { Target } \\
\text { reassignment: } \\
\text { physiologic/ } \\
\text { Targeted muscle } \\
\text { reinnervation }\end{array}$ & $\begin{array}{l}\text { To review the } \\
\text { current literature } \\
\text { on the treatment } \\
\text { of terminal } \\
\text { neuromas }\end{array}$ & $\begin{array}{l}\text { Comprehensive } \\
\text { literature review }\end{array}$ & 98 Articles cited & $\begin{array}{l}4 \text { Treatment } \\
\text { categories } \\
\text { formulated with } \\
\text { report and } \\
\text { interpretation of } \\
\text { data }\end{array}$ & $\begin{array}{l}4 \text { Categories include } \\
\text { epineural closure; } \\
\text { nerve transposition } \\
\text { with implantation; } \\
\text { neurorrhaphy, and } \\
\text { alternate target } \\
\text { reinnervation. } \\
\text { Minimal } \\
\text { quantitative } \\
\text { evidence regarding } \\
\text { prevention given }\end{array}$ & $\begin{array}{l}\text { Well organized, } \\
\text { thorough review }\end{array}$ & $\begin{array}{l}\text { No disclosure of } \\
\text { article selection } \\
\text { protocol in primary } \\
\text { publication, } \\
\text { prevention of } \\
\text { neuromas or } \\
\text { phantom limb pain } \\
\text { was not purpose of } \\
\text { review }\end{array}$ \\
\hline $\begin{array}{l}\text { Kuiken } 2017 \\
\text { [72] }\end{array}$ & $\begin{array}{l}\text { Target } \\
\text { reassignment: } \\
\text { physiologic/ } \\
\text { Targeted muscle } \\
\text { reinnervation }\end{array}$ & $\begin{array}{l}\text { To present the } \\
\text { technique of } \\
\text { TMR in upper } \\
\text { and lower } \\
\text { extremity } \\
\text { amputations }\end{array}$ & $\begin{array}{l}\text { Technique } \\
\text { presentation }\end{array}$ & 100 Patients & $\begin{array}{l}\text { Presentation of } \\
\text { methods, } \\
\text { experience and } \\
\text { literature }\end{array}$ & $\begin{array}{l}1 \text { of } 100 \text { patients } \\
\text { who underwent } \\
\text { TMR were re- } \\
\text { explored to resect } \\
\text { a neuroma. }\end{array}$ & $\begin{array}{l}\text { Well organized, } \\
\text { adhere to strong } \\
\text { surgical } \\
\text { principles }\end{array}$ & $\begin{array}{l}\text { Neuroma presentation } \\
\text { a secondary topic, } \\
\text { no statistical } \\
\text { analysis, minimal } \\
\text { presentation of raw } \\
\text { data, assessor bias }\end{array}$ \\
\hline $\begin{array}{l}\text { Alexander } \\
2019 \text { [73] }\end{array}$ & $\begin{array}{l}\text { Target } \\
\text { reassignment: } \\
\text { physiologic/ } \\
\text { Targeted muscle } \\
\text { reinnervation }\end{array}$ & $\begin{array}{l}\text { To assess TNPLP } \\
\text { specifically in } \\
\text { patients who } \\
\text { undergo } \\
\text { oncologic } \\
\text { amputation }\end{array}$ & $\begin{array}{l}\text { Single institution } \\
\text { cohort study }\end{array}$ & $\begin{array}{l}27 \text { Patients } \\
\text { underwent } \\
\text { oncologic } \\
\text { amputation at } \\
\text { a single } \\
\text { institution and } \\
\text { were } \\
\text { compared to } \\
58 \text { patients } \\
\text { treated at } \\
\text { other } \\
\text { institutions }\end{array}$ & $\begin{array}{l}\text { PROMIS looking at } \\
\text { pain intensity, pain } \\
\text { behavior, and pain } \\
\text { interference }\end{array}$ & $\begin{array}{l}\text { Mean differences in } \\
\text { PROMIS scores for } \\
\text { TNPLP were } 5.855 \\
(P=0.15), 5.896 \\
(P=0.033), 7.435 \\
(P=0.011) \text { for } \\
\text { pain intensity, pain } \\
\text { behavior and pain } \\
\text { interference, } \\
\text { respectively. } \\
\text { Mean differences in } \\
\text { PROMIS scores for } \\
\text { residual limb pain } \\
\text { were 5.477 } \\
(P=0.031), 6.195 \\
(P=0.028), 6.816 \\
(P=0.014) \text { for } \\
\text { pain intensity, pain } \\
\text { behavior and pain } \\
\text { interference, } \\
\text { respectively. }\end{array}$ & $\begin{array}{l}\text { Prospective, } \\
\text { utilization of } \\
\text { standardized } \\
\text { pain related } \\
\text { measures }\end{array}$ & $\begin{array}{l}\text { Nonrandomized, } \\
\text { nonblinded, } \\
\text { multimodal } \\
\text { approach to } \\
\text { postoperative pain } \\
\text { control could a } \\
\text { confounder, of } 27 \\
\text { patients who } \\
\text { underwent TMR, } \\
\text { only } 15 \text { had follow } \\
\text { up greater than } 1 \\
\text { year despite median } \\
\text { follow-up of } 16 \\
\text { months }\end{array}$ \\
\hline $\begin{array}{l}\text { Bowen } 2019 \\
\text { [74] }\end{array}$ & $\begin{array}{l}\text { Target } \\
\text { reassignment: } \\
\text { physiologic/ } \\
\text { Targeted muscle } \\
\text { reinnervation }\end{array}$ & $\begin{array}{l}\text { To present results } \\
\text { of TMR in BKA } \\
\text { as a means of } \\
\text { preventing } \\
\text { TNPLP }\end{array}$ & Case series & $\begin{array}{l}22 \text { Patients } \\
\text { (18 primary, } \\
4 \text { secondary) }\end{array}$ & $\begin{array}{l}\text { Physical exam, } \\
\text { subjective patient } \\
\text { reporting }\end{array}$ & $\begin{array}{l}72 \% \text { of the primary } \\
\text { TMR cohort } \\
\text { experience } \\
\text { phantom limb pain } \\
\text { in the first month, } \\
\text { with a decline to } \\
19 \% \text { at } 3 \text { months, } \\
\text { and } 13 \% \text { at } 6 \\
\text { months. }\end{array}$ & 1 Year follow up & $\begin{array}{l}\text { Nonrandomized, } \\
\text { nonblinded, data } \\
\text { specific to BKA }\end{array}$ \\
\hline
\end{tabular}


Table 1. Continued

\begin{tabular}{|c|c|c|c|c|c|c|c|c|}
\hline Author & $\begin{array}{l}\text { Category/ } \\
\text { Technique }\end{array}$ & Purpose & Design & Sample size & $\begin{array}{l}\text { Analysis } \\
\text { techniques }\end{array}$ & Quantitative results & Strengths & Limitations \\
\hline $\begin{array}{l}\text { Valerio } 2019 \\
\text { [75] }\end{array}$ & $\begin{array}{l}\text { Target } \\
\text { reassignment: } \\
\text { physiologic/ } \\
\text { Targeted muscle } \\
\text { reinnervation }\end{array}$ & $\begin{array}{l}\text { To assess if TMR } \\
\text { at the time of } \\
\text { amputation } \\
\text { decreases } \\
\text { incidence and } \\
\text { severity of } \\
\text { TNPLP }\end{array}$ & $\begin{array}{l}\text { Multi-institutional } \\
\text { cohort }\end{array}$ & $\begin{array}{l}51 \text { Patients } \\
\text { compared } \\
\text { with } 438 \\
\text { major limb } \\
\text { amputees }\end{array}$ & $\begin{array}{l}11 \text { NRS and PROMIS } \\
\text { looking at pain, } \\
\text { intensity, behavior, } \\
\text { and interference }\end{array}$ & $\begin{array}{l}\text { TMR showed lower } \\
\text { median PROMIS } \\
\text { t-scores for TNPLP } \\
\text { with pain behavior } \\
(P<0.001) \text {, pain } \\
\text { intensity } \\
(P<0.001) \text { and } \\
\text { pain interference } \\
(P<0.001) \text {. A } \\
\text { similar pattern was } \\
\text { seen with residual } \\
\text { pain in regards to } \\
\text { pain behavior } \\
(P<0.001) \text {, pain } \\
\text { intensity } \\
(P<0.001) \text { and } \\
\text { pain interference } \\
(P<0.001) \text {. }\end{array}$ & $\begin{array}{l}\text { Prospective, } \\
\text { utilization of } \\
\text { standardized } \\
\text { pain related } \\
\text { measures }\end{array}$ & $\begin{array}{l}\text { Non-randomized, } \\
\text { nonblinded, } \\
\text { referrals for } \\
\text { amputee group at } \\
\text { discretion of } \\
\text { surgical team } \\
\text { performing } \\
\text { amputation, } \\
\text { 3-month minimum } \\
\text { follow-up time }\end{array}$ \\
\hline $\begin{array}{l}\text { Kubiak } 2019 \\
\text { [66] }\end{array}$ & $\begin{array}{l}\text { Target } \\
\text { reassignment: } \\
\text { physiologic/ } \\
\text { RPNI }\end{array}$ & $\begin{array}{l}\text { To present results } \\
\text { of creation of } \\
\text { RPNI as a means } \\
\text { of preventing } \\
\text { TNPLP and } \\
\text { neuromas }\end{array}$ & Retrospective & $\begin{array}{l}45 \text { Patients } \\
\text { underwent } \\
\text { RPNI matched } \\
\text { to } 45 \text { control } \\
\text { patients } \\
\text { selected from } \\
\text { a pool of } 178\end{array}$ & $\begin{array}{l}\text { Documentation of } \\
\text { physical exam } \\
\text { findings and } \\
\text { patient reporting. } \\
\text { Fischer exact test } \\
\end{array}$ & $\begin{array}{l}\text { No symptomatic } \\
\text { neuromas noted in } \\
\text { the intervention } \\
\text { group ( } 0 \% \text { vs. } \\
13.3 \%, \mathrm{P}=0.026) \text {. } \\
\text { There was a } \\
\text { reduced incidence } \\
\text { of TNPLP in the } \\
\text { intervention group } \\
\text { (51.1\% vs. } 91.1 \% \text {, } \\
P<0.0001) \text {. }\end{array}$ & $\begin{array}{l}\text { Long mean } \\
\text { duration of } \\
\text { follow-up on } \\
\text { average of } 1 \\
\text { year }\end{array}$ & $\begin{array}{l}\text { Inconsistence chart } \\
\text { documentation }\end{array}$ \\
\hline $\begin{array}{l}\text { De Smet } \\
1996 \text { [68] }\end{array}$ & $\begin{array}{l}\text { Target in } \\
\text { continuity/ } \\
\text { Preservation of } \\
\text { continuity }\end{array}$ & $\begin{array}{l}\text { To present results } \\
\text { of mid-finger } \\
\text { amputation } \\
\text { reconstructions } \\
\text { with bi- } \\
\text { neurovascular } \\
\text { bundle pedicled } \\
\text { volar pulp flaps }\end{array}$ & Case series & 4 Patients & $\begin{array}{l}\text { Personal experience } \\
\text { and opinion }\end{array}$ & $\begin{array}{l}\text { All } 4 \text { flaps survived } \\
\text { with sensibility } \\
\text { equal to } \\
\text { preoperative }\end{array}$ & $\begin{array}{l}\text { May be beneficial } \\
\text { when indicated }\end{array}$ & $\begin{array}{l}\text { Nonrandomized, } \\
\text { nonblinded, small } \\
\text { sample size, no } \\
\text { comparison. } \\
\text { minimal long-term } \\
\text { follow-up, assessor } \\
\text { bias }\end{array}$ \\
\hline $\begin{array}{l}\text { St-Laurent } \\
1996 \text { [69] }\end{array}$ & $\begin{array}{l}\text { Target in } \\
\text { continuity/ } \\
\text { Preservation of } \\
\text { continuity }\end{array}$ & $\begin{array}{l}\text { To assess results } \\
\text { of elective digital } \\
\text { amputation with } \\
\text { bipedicled } \\
\text { neurovascular } \\
\text { volar pulp flap }\end{array}$ & Case series & $\begin{array}{l}8 \text { Patients, } 9 \\
\text { amputations }\end{array}$ & $\begin{array}{l}\text { Physical exam, } \\
\text { patient reporting }\end{array}$ & $\begin{array}{l}7 \text { Patients without } \\
\text { painful neuroma, } \\
1 \text { patient with } \\
\text { preoperative pain } \\
\text { that did not } \\
\text { improve } \\
\text { postoperatively. }\end{array}$ & Prospective & $\begin{array}{l}\text { Minimal long-term } \\
\text { follow-up (range, } \\
\text { 2-9 months), small } \\
\text { sample size }\end{array}$ \\
\hline
\end{tabular}

ANOVA, analysis of variance; BKA, below-knee amputation; CCU, centro-central union (the direct union of two nerve endings, or the splitting and union of a single nerve ending); NRS, numerical rating scale; PROMIS, Patient-Reported Outcomes Measurement Information System; RPNI, regenerative peripheral nerve interface (reinnervated, nonvascularized muscle grafts; TMR, targeted muscle reinnervation (the coaptation of mixed nerves into terminal motor nerves for the purpose of creating functional electromyographic signals; TNI, targeted nerve implantation (coaptation of nerves solely for the purpose of neuroma prevention); TNPLP, terminal neuroma or phantom limb pain; S2PD, static two-point discrimination; VAS, visual analog scale.

$(\mathrm{n}=7)$, followed by RPNI $(\mathrm{n}=1)$ and TNI $(\mathrm{n}=1)$.

Souza et al. (2014) [19] performed a retrospective chart review evaluating the effect of TMR on 11 patients without terminal neuroma and 15 patients with established terminal neuroma. None of the 11 patients who presented without neuroma developed a neuroma after TMR.

Bowen et al. (2017) [6] performed a comprehensive literature review and presentation of their experience with TMR as a reli- able treatment for TNPLP. In a further prospective study in 2019 by Bowen et al. [74], 22 patients underwent TMR at the time of below-knee amputation and none of the patients reported neuroma pain postoperatively. While no numerical measures were provided, the authors disclosed that a clinical trial underway at their institution showed encouraging results of TMR for the prevention of TNPLP.

Ives et al. (2017) [11] performed a comprehensive literature 
review on the treatment of terminal neuroma. These authors developed the categorization system that was modified for use in this review. Their study investigated TMR and RPNI. Both techniques were described as promising procedures to reduce the incidence of terminal neuroma.

Kuiken et al. (2017) [72] presented methods, experiences and a review of the literature on TMR in upper and lower extremity amputation. One hundred patients were analyzed, and only one was re-explored for neuroma resection. While neuroma prevention was a secondary topic and no statistical analysis was presented, the authors mentioned TMR as a promising, albeit experimental, technique for prevention of terminal neuroma.

Alexander et al. (2019) [73] performed a prospective study assessing TNPLP among patients who specifically underwent TMR with concurrent oncologic amputations. The study measured post-amputation pain using the Patient-Reported Outcomes Measurement Information System (PROMIS) and evaluated pain intensity, pain behavior, and pain interference. $\mathrm{Al}$ though the article did not include specific surgical techniques, the authors referenced prior works [76-78]. The authors demonstrated that patients who underwent concurrent TMR had lower incidence rates of TNPLP and residual limb pain than patients who did not undergo concurrent TMR. The mean differences in PROMIS scores for TNPLP were $5.855(\mathrm{P}=0.15)$, $5.896(\mathrm{P}=0.033)$, and $7.435(\mathrm{P}=0.011)$ for pain intensity, pain behavior, and pain interference, respectively. Meanwhile, the mean differences in PROMIS scores for residual limb pain were $5.477(\mathrm{P}=0.031), 6.195(\mathrm{P}=0.028), 6.816(\mathrm{P}=0.014)$ for pain intensity, pain behavior, and pain interference, respectively.

Similarly, Valerio et al. (2019) [75] performed a prospective study assessing TNPLP in patients who underwent TMR at the time of amputation versus those who only had amputation using the PROMIS scoring system. Their study demonstrated that those who underwent concurrent TMR had significantly lower median PROMIS $\mathrm{t}$-scores $(\mathrm{P}<0.001)$ for TNPLP with regard to pain behavior, pain intensity, and pain interference. Likewise, a similar pattern was seen with residual pain in regard to pain behavior, pain intensity, and pain interference.

The only study examining the prevention of TNPLP using RPNI at the time of amputation was that by Kubiak et al. in 2019 [66]. The authors reported a significantly lower incidence of postoperative TNPLP and symptomatic neuroma in this retrospective study. Specifically, the authors found that six control patients developed symptomatic neuroma, while the TMR group had no symptomatic neuroma cases $(\mathrm{P}=0.026)$. Furthermore, 41 control patients reported TNPLP, whereas only 23 patients in the TMR group did so $(\mathrm{P}<0.0001)$.

Pet et al. (2014) [15] performed a retrospective chart review to investigate whether TNI prevents neuroma formation in acute traumatic amputation. Eleven of 12 patients were free of palpable neuroma pain. Despite the retrospective nature of this study, the authors concluded that TNI is a promising technique for the prevention of terminal neuroma.

\section{Target in continuity}

There were only two older studies (published in 1996) addressing the efficacy of preservation of continuity in TNPLP prevention $[68,69]$. The indications for this technique are limited to pathology that necessitates segmental amputation of a limb, but spares intervening neurovascular tissues. When the intermediate extremity is amputated and the proximal and distal ends are reattached with their neurovascular connections intact, the intact nerves should provide no opportunity for the development of TNPLP.

A case series of four patients who underwent neurovascular bundle pedicled volar pulp flaps for mid-finger amputation was presented by De Smet in 1996 [68]. All four flaps survived with sensibility equal to the preoperative status and no neuroma formation.

In the same year, St-Laurent and Duclos [69] presented a case series of eight patients who underwent the same procedure. Seven of those eight patients did not develop painful neuroma. The patient who reported pain suffered from preoperative pain that did not improve after surgery. We have termed this technique "preservation of continuity."

\section{DISCUSSION}

Post-amputation TNPLP results in significant patient disability and an economic burden on the healthcare system. Although a myriad of treatment strategies, both non-surgical and surgical, have been studied and implemented, their success has been limited. In contrast, the surgical prevention of TNPLP at the time of primary amputation, although it has not been well defined in the literature, has shown the greatest potential for reducing the incidence of this debilitating condition.

To our knowledge, this is the first literature review of the surgical prevention of TNPLP and the first attempt at formulating a target-based algorithm to describe the current trends (Fig. 3). Despite the paucity of high-quality literature on the surgical prevention of TNPLP, all studies have shown promising results and affirmed the need for a more vigorous investigation of this paradigm.

\section{Target deficient}

End closure may provide a simpler technique; however, it does 


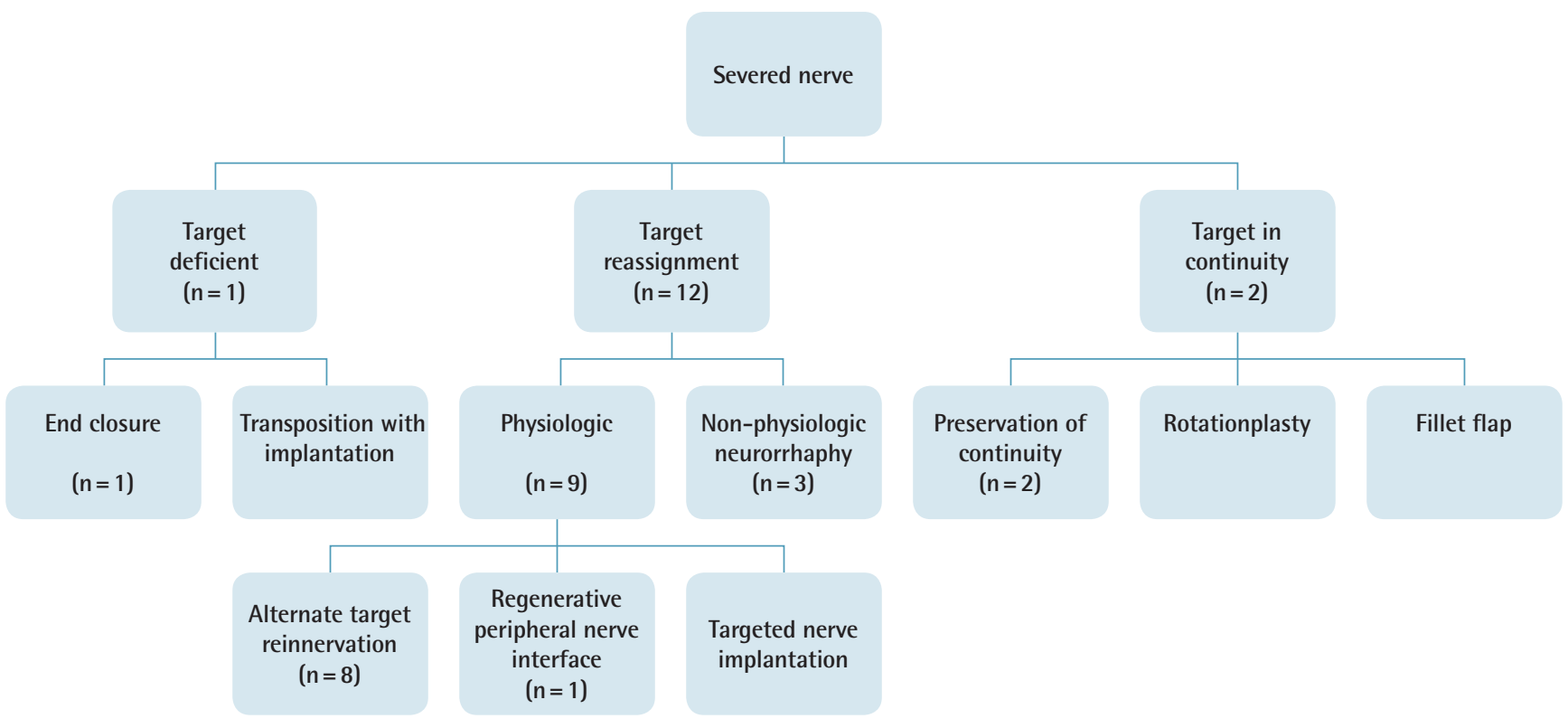

Fig. 3. A target-based algorithm for terminal neuroma and phantom limb pain surgical prevention terminology.

not follow the theoretical trends towards providing an end organ for nerve ingrowth and does not have strong support from the literature. Transposition with implantation into bone, muscle, or fascia is a well described and widely utilized method for the treatment of established neuroma [7,79-83]. Surprisingly, this literature review identified no studies evaluating the use of transposition and implantation in the primary, preventative setting. Future studies on transposition with implantation would be a logical step towards the pursuit of an efficient, effective strategy to prevent TNPLP.

\section{Physiologic target reassignment}

Within the last 6 years, a rapid proliferation in information on TMR, TNI, and RPNI reflects momentum in this area, especially TMR. Multiple high-quality respective cohort studies utilizing standardized pain scales (NRS [numerical rating scale] and PROMIS) have demonstrated statistically significant superiority of TMR and TNI for surgical prophylaxis against TNPLP over no nerve interventions [73-75].

\section{Non-physiologic target reassignment}

According to our review, neurorrhaphy has demonstrated good outcomes and should be considered in most cases when more complex techniques are unable to be performed. While this technique has a moderate learning curve, it can be performed under loupe magnification and, once routine, may be performed quickly. Future prospective trials may confirm the results of the current, low-powered studies $[9,67,71]$.

\section{Target in continuity}

This review did not identify any studies evaluating the incidence of TNPLP after rotationplasty or fillet flap, both of which have been shown to improve quality of life, prosthetic usage, functional outcome, and emotional well-being in oncologic patients [84-95].

Our 15 studies spanned 3 decades, indicating plastic surgeons' early recognition of the debilitating impact of TNPLP on patient quality of life and, consequently, the need for prevention. Earlier studies emphasized end closure, preservation of continuity, and neurorrhaphy/CCU, whereas TMR has been the mainstay of interventions in the past 5 years. As expected, early studies were mainly case series, technique reviews, and retrospective reviews of single-surgeon experiences, whereas later studies were prospective trials involving multiple surgeons and sometimes institutions. The later trials also employed more standardized data reporting tools, as well as validated patient-centered variables such as the PROMIS score.

Our algorithm maintains previous terminology and organizes each strategy into target-based categories. This may help to guide future academic and clinical analyses of the topic. This target-based algorithm may also guide treatment based on each patient's individual anatomy and the nerve targets that the patient offers.

\section{CONCLUSIONS}

In conclusion, this literature review demonstrates a small, yet growing body of literature on the surgical prophylaxis of TN- 
PLP. The strongest body of literature favors the physiologic target reassignment techniques of TMR and TNI. Neurorrhaphy and transposition with implantation are supported by less robust evidence, but merit future study as simpler alternatives.

\section{NOTES}

\section{Conflict of interest}

No potential conflict of interest relevant to this article was reported.

\section{Author contribution}

Conceptualization: RN Bogdasarian, SB Cai, A Ignatiuk, ES Lee. Data curation: RN Bogdasarian, BNN Tran. Formal analysis: RN Bogdasarian, SB Cai, BNN Tran. Methodology: RN Bogdasarian, SB Cai, A Ignatiuk, ES Lee. Project administration: RN Bogdasarian, SB Cai. Visualization: RN Bogdasarian, SB Cai, BNN Tran. Writing - original draft: RN Bogdasarian. Writing - review \& editing: RN Bogdasarian, BNN Tran.

\section{ORCID}

Steven B. Cai https://orcid.org/0000-0002-2522-3337

Bao Ngoc N. Tran https://orcid.org/0000-0003-4301-1407

\section{REFERENCES}

1. Narres M, Kvitkina T, Claessen H, et al. Incidence of lower extremity amputations in the diabetic compared with the non-diabetic population: a systematic review. PLoS One 2017;12:e0182081.

2. Bosmans JC, Geertzen JH, Post WJ, et al. Factors associated with phantom limb pain: a 31/2-year prospective study. Clin Rehabil 2010;24:444-53.

3. Hanley MA, Jensen MP, Smith DG, et al. Preamputation pain and acute pain predict chronic pain after lower extremity amputation.J Pain 2007;8:102-9.

4. Chen MC, Lee SS, Hsieh YL, et al. Influencing factors of outcome after lower-limb amputation: a five-year review in a plastic surgical department. Ann Plast Surg 2008;61:3148.

5. Barbera J, Albert-Pamplo R. Centrocentral anastomosis of the proximal nerve stump in the treatment of painful amputation neuromas of major nerves. J Neurosurg 1993;79:3314.

6. Bowen JB, Wee CE, Kalik J, et al. Targeted muscle reinnervation to improve pain, prosthetic tolerance, and bioprosthetic outcomes in the amputee. Adv Wound Care (New Rochelle) 2017;6:261-7.
7. Dellon AL, Mackinnon SE, Pestronk A. Implantation of sensory nerve into muscle: preliminary clinical and experimental observations on neuroma formation. Ann Plast Surg 1984;12:30-40.

8. Ducic I, Mesbahi AN, Attinger CE, et al. The role of peripheral nerve surgery in the treatment of chronic pain associated with amputation stumps. Plast Reconstr Surg 2008;121: 908-14.

9. Gorkisch K, Boese-Landgraf J, Vaubel E. Treatment and prevention of amputation neuromas in hand surgery. Plast Reconstr Surg 1984;73:293-9.

10. Halbert J, Crotty M, Cameron ID. Evidence for the optimal management of acute and chronic phantom pain: a systematic review. Clin J Pain 2002;18:84-92.

11. Ives GC, Kung TA, Nghiem BT, et al. Current state of the surgical treatment of terminal neuromas. Neurosurgery 2018;83:354-64.

12. Martins RS, Siqueira MG, Heise CO, et al. Interdigital direct neurorrhaphy for treatment of painful neuroma due to finger amputation. Acta Neurochir (Wien) 2015;157:667-71.

13. Moesker AA, Karl HW, Trescot AM. Treatment of phantom limb pain by cryoneurolysis of the amputated nerve. Pain Pract 2014;14:52-6.

14. Patrick J, Frank W, Theodora M, et al. The pedicled serratus anterior muscle wrap-around flap: a treatment option in the management of posttraumatic axillary neuroma and neuropathic pain. Ann Plast Surg 2010;65:170-3.

15. Pet MA, Ko JH, Friedly JL, et al. Does targeted nerve implantation reduce neuroma pain in amputees? Clin Orthop Relat Res 2014;472:2991-3001.

16. Pet MA, Ko JH, Friedly JL, et al. Traction neurectomy for treatment of painful residual limb neuroma in lower extremity amputees. J Orthop Trauma 2015;29:e321-5.

17. Prantl L, Schreml S, Heine N, et al. Surgical treatment of chronic phantom limb sensation and limb pain after lower limb amputation. Plast Reconstr Surg 2006;118:1562-72.

18. Slooff AC. Microsurgical possibilities in the treatment of peripheral pain. Clin Neurol Neurosurg 1977;80:107-11.

19. Souza JM, Cheesborough JE, Ko JH, et al. Targeted muscle reinnervation: a novel approach to postamputation neuroma pain. Clin Orthop Relat Res 2014;472:2984-90.

20. Swanson AB, Boeve NR, Lumsden RM. The prevention and treatment of amputation neuromata by silicone capping. J Hand Surg Am 1977;2:70-8.

21. Woo SL, Kung TA, Brown DL, et al. Regenerative peripheral nerve interfaces for the treatment of postamputation neuroma pain: a pilot study. Plast Reconstr Surg Glob Open 2016;4:e1038. 
22. Wood MR, Hunter GA, Millstein SG. The value of revision surgery after initial amputation of an upper or lower limb. Prosthet Orthot Int 1987;11:17-20.

23. Wood VE, Mudge MK. Treatment of neuromas about a major amputation stump. J Hand Surg Am 1987;12:302-6.

24. Goldstein SA, Sturim HS. Intraosseous nerve transposition for treatment of painful neuromas. J Hand Surg Am 1985; 10:270-4.

25. Jensen TS, Krebs B, Nielsen J, et al. Phantom limb, phantom pain and stump pain in amputees during the first 6 months following limb amputation. Pain 1983;17:243-56.

26. Jensen TS, Krebs B, Nielsen J, et al. Immediate and longterm phantom limb pain in amputees: incidence, clinical characteristics and relationship to pre-amputation limb pain. Pain 1985;21:267-78.

27. Nikolajsen L, Ilkjaer S, Jensen TS. Effect of preoperative extradural bupivacaine and morphine on stump sensation in lower limb amputees. Br J Anaesth 1998;81:348-54.

28. Mioton LM, Dumanian GA. Targeted muscle reinnervation and prosthetic rehabilitation after limb loss. J Surg Oncol 2018;118:807-14.

29. Andoh J, Milde C, Tsao JW, et al. Cortical plasticity as a basis of phantom limb pain: fact or fiction? Neuroscience 2018;387:85-91.

30. Dettmers C, Adler T, Rzanny R, et al. Increased excitability in the primary motor cortex and supplementary motor area in patients with phantom limb pain after upper limb amputation. Neurosci Lett 2001;307:109-12.

31. Flor H. Phantom-limb pain: characteristics, causes, and treatment. Lancet Neurol 2002;1:182-9.

32. Flor H. Cortical reorganisation and chronic pain: implications for rehabilitation. J Rehabil Med 2003;(41 Suppl): 6672.

33. Flor H. Maladaptive plasticity, memory for pain and phantom limb pain: review and suggestions for new therapies. Expert Rev Neurother 2008;8:809-18.

34. Flor H, Birbaumer N. Phantom limb pain: cortical plasticity and novel therapeutic approaches. Curr Opin Anaesthesiol 2000;13:561-4.

35. Flor H, Diers M, Andoh J. The neural basis of phantom limb pain. Trends Cogn Sci 2013; 17:307-8.

36. Flor H, Nikolajsen L, Staehelin Jensen T. Phantom limb pain: a case of maladaptive CNS plasticity? Nat Rev Neurosci 2006;7:873-81.

37. Foell J, Andoh J, Bekrater-Bodmann R, et al. Peripheral origin of phantom limb pain: is it all resolved? Pain 2014;155: 2205-6.

38. Grusser SM, Winter C, Muhlnickel W, et al. The relation- ship of perceptual phenomena and cortical reorganization in upper extremity amputees. Neuroscience 2001;102:26372.

39. Karl A, Birbaumer N, Lutzenberger W, et al. Reorganization of motor and somatosensory cortex in upper extremity amputees with phantom limb pain. J Neurosci 2001;21:360918.

40. Knecht S, Henningsen $H$, Elbert T, et al. Cortical reorganization in human amputees and mislocalization of painful stimuli to the phantom limb. Neurosci Lett 1995;201:2624.

41. Makin TR, Scholz J, Henderson Slater D, et al. Reassessing cortical reorganization in the primary sensorimotor cortex following arm amputation. Brain 2015;138(Pt 8):2140-6.

42. Montoya P, Ritter K, Huse E, et al. The cortical somatotopic map and phantom phenomena in subjects with congenital limb atrophy and traumatic amputees with phantom limb pain. Eur J Neurosci 1998;10:1095-102.

43. Oouchida Y, Sudo T, Inamura T, et al. Maladaptive change of body representation in the brain after damage to central or peripheral nervous system. Neurosci Res 2016;104:3843.

44. Raffin E, Richard N, Giraux P, et al. Primary motor cortex changes after amputation correlate with phantom limb pain and the ability to move the phantom limb. Neuroimage 2016;130:134-44.

45. Reilly KT, Sirigu A. The motor cortex and its role in phantom limb phenomena. Neuroscientist 2008; 14:195-202.

46. Wu CW, Kaas JH. The effects of long-standing limb loss on anatomical reorganization of the somatosensory afferents in the brainstem and spinal cord. Somatosens Mot Res 2002; 19:153-63.

47. Yanagisawa T, Fukuma R, Seymour B, et al. Induced sensorimotor brain plasticity controls pain in phantom limb patients. Nat Commun 2016;7:13209.

48. Andreae MH, Andreae DA. Local anaesthetics and regional anaesthesia for preventing chronic pain after surgery. Cochrane Database Syst Rev 2012;10:CD007105.

49. Borghi B, D'Addabbo M, White PF, et al. The use of prolonged peripheral neural blockade after lower extremity amputation: the effect on symptoms associated with phantom limb syndrome. Anesth Analg 2010;111:1308-15.

50. Enneking FK, Scarborough MT, Radson EA. Local anesthetic infusion through nerve sheath catheters for analgesia following upper extremity amputation. Clinical report. Reg Anesth 1997;22:351-6.

51. Finsen V, Persen L, Lovlien M, et al. Transcutaneous electrical nerve stimulation after major amputation. J Bone Joint 
Surg Br 1988;70:109-12.

52. Fisher A, Meller Y. Continuous postoperative regional analgesia by nerve sheath block for amputation surgery: a pilot study. Anesth Analg 1991;72:300-3.

53. Grant AJ, Wood C. The effect of intra-neural local anaesthetic infusion on pain following major lower limb amputation. Scott Med J 2008;53:4-6.

54. Hanling SR, Wallace SC, Hollenbeck KJ, et al. Preamputation mirror therapy may prevent development of phantom limb pain: a case series. Anesth Analg 2010;110:611-4.

55. Hayes C, Armstrong-Brown A, Burstal R. Perioperative intravenous ketamine infusion for the prevention of persistent post-amputation pain: a randomized, controlled trial. Anaesth Intensive Care 2004;32:330-8.

56. Jahangiri M, Jayatunga AP, Bradley JW, et al. Prevention of phantom pain after major lower limb amputation by epidural infusion of diamorphine, clonidine and bupivacaine. Ann R Coll Surg Engl 1994;76:324-6.

57. Lambert Aw, Dashfield Ak, Cosgrove C, et al. Randomized prospective study comparing preoperative epidural and intraoperative perineural analgesia for the prevention of postoperative stump and phantom limb pain following major amputation. Reg Anesth Pain Med 2001;26:316-21.

58. Madabhushi L, Reuben SS, Steinberg RB, et al. The efficacy of postoperative perineural infusion of bupivacaine and clonidine after lower extremity amputation in preventing phantom limb and stump pain. J Clin Anesth 2007;19:2269.

59. Morey TE, Giannoni J, Duncan E, et al. Nerve sheath catheter analgesia after amputation. Clin Orthop Relat Res 2002; (397):281-9.

60. Mulvey MR, Bagnall AM, Johnson MI, et al. Transcutaneous electrical nerve stimulation (TENS) for phantom pain and stump pain following amputation in adults. Cochrane Database Syst Rev 2010;(5):CD007264.

61. Ong BY, Arneja A, Ong EW. Effects of anesthesia on pain after lower-limb amputation.J Clin Anesth 2006;18:600-4.

62. Pavy TJ, Doyle DL. Prevention of phantom limb pain by infusion of local anaesthetic into the sciatic nerve. Anaesth Intensive Care 1996;24:599-600.

63. Pinzur MS, Garla PG, Pluth T, et al. Continuous postoperative infusion of a regional anesthetic after an amputation of the lower extremity. A randomized clinical trial. J Bone Joint Surg Am 1996;78:1501-5.

64. Wilson JA, Nimmo AF, Fleetwood-Walker SM, et al. A randomised double blind trial of the effect of pre-emptive epidural ketamine on persistent pain after lower limb amputation. Pain 2008;135:108-18.
65. Wu CL, Tella P, Staats PS, et al. Analgesic effects of intravenous lidocaine and morphine on postamputation pain: a randomized double-blind, active placebo-controlled, crossover trial. Anesthesiology 2002;96:841-8.

66. Kubiak CA, Kemp SWP, Cederna PS, et al. Prophylactic regenerative peripheral nerve interfaces to prevent postamputation pain. Plast Reconstr Surg 2019;144:421e-430e.

67. Belcher HJ, Pandya AN. Centro-central union for the prevention of neuroma formation after finger amputation. J Hand Surg Br 2000;25:154-9.

68. De Smet L. Preventing neuroma formation in finger amputation. Acta Orthop Belg 1996;62:69-70.

69. St-Laurent JY, Duclos L. Prevention of neuroma in elective digital amputations by utilization of neurovascular island flap. Ann Chir Main Memb Super 1996;15:50-4.

70. Yuksel F, Kislaoglu E, Durak N, et al. Prevention of painful neuromas by epineural ligatures, flaps and grafts. Br J Plast Surg 1997;50:182-5.

71. Economides JM, DeFazio MV, Attinger CE, et al. Prevention of painful neuroma and phantom limb pain after transfemoral amputations through concomitant nerve coaptation and collagen nerve wrapping. Neurosurgery 2016;79:50813.

72. Kuiken TA, Barlow AK, Hargrove L, et al. Targeted muscle reinnervation for the upper and lower extremity. Tech Orthop 2017;32:109-16.

73. Alexander JH, Jordan SW, West JM, et al. Targeted muscle reinnervation in oncologic amputees: early experience of a novel institutional protocol. J Surg Oncol 2019;120:348-58.

74. Bowen JB, Ruter D, Wee C, et al. Targeted muscle reinnervation technique in below-knee amputation. Plast Reconstr Surg 2019;143:309-12.

75. Valerio IL, Dumanian GA, Jordan SW, et al. Preemptive treatment of phantom and residual limb pain with targeted muscle reinnervation at the time of major limb amputation. J Am Coll Surg 2019;228:217-26.

76. Agnew SP, Schultz AE, Dumanian GA, et al. Targeted reinnervation in the transfemoral amputee: a preliminary study of surgical technique. Plast Reconstr Surg 2012;129:187-94.

77. Gart MS, Souza JM, Dumanian GA. Targeted muscle reinnervation in the upper extremity amputee: a technical roadmap. J Hand Surg Am 2015;40:1877-88.

78. Morgan EN, Kyle Potter B, Souza JM, et al. Targeted muscle reinnervation for transradial amputation: description of operative technique. Tech Hand Up Extrem Surg 2016;20:16671.

79. Prasetyono TO, Permatasari E, Soetrisno E. Implantation of nerve stump inside a vein and a muscle: comparing neuro- 
ma formation in rat. Int Surg 2014;99:807-11.

80. Lewin-Kowalik J, Marcol W, Kotulska K, et al. Prevention and management of painful neuroma. Neurol Med Chir (Tokyo) 2006;46:62-7.

81. Low CK, Chew SH, Song IC, et al. Implantation of a nerve ending into a vein. Clin Orthop Relat Res 2000;(379):2426.

82. Otfinowski J, Pawelec A, Kaluza J. Implantation of peripheral neural stump into muscle and its effect on the development of posttraumatic neuroma. Pol J Pathol 1994;45:195202.

83. Zhang B, Guo Y, Liang Y. Experimental study on prevention and treatment of neuroma by implanting nerve stump into muscle. Zhongguo Xiu Fu Chong Jian Wai Ke Za Zhi 1997; 11:325-7.

84. Kim PS, Ko JH, O'Shaughnessy KK, et al. The effects of targeted muscle reinnervation on neuromas in a rabbit rectus abdominis flap model. J Hand Surg Am 2012;37:1609-16.

85. Ko JH, Kim PS, Smith DG. Targeted muscle reinnervation as a strategy for neuroma prevention. In: Kuiken TA, Schultz Feuser AE, Barlow AK, editors. Targeted muscle reinnervation: a neural interface for artificial limbs (Series in Medical Physics and Biomedical Engineering). Boca Raton: Taylor \& Francis; 2013. p. 45-66.

86. Chen A, Yao J, Kuiken T, et al. Cortical motor activity and reorganization following upper-limb amputation and subsequent targeted reinnervation. Neuroimage Clin 2013;3:498506.

87. Gradl G, Postl LK, Lenze U, et al. Long-term functional out- come and quality of life following rotationplasty for treatment of malignant tumors. BMC Musculoskelet Disord 2015;16:262.

88. Forni C, Gaudenzi N, Zoli M, et al. Living with rotationplasty: quality of life in rotationplasty patients from childhood to adulthood. J Surg Oncol 2012;105:331-6.

89. Fuchs B, Kotajarvi BR, Kaufman KR, et al. Functional outcome of patients with rotationplasty about the knee. Clin Orthop Relat Res 2003; (415):52-8.

90. Furtado S, Grimer RJ, Cool P, et al. Physical functioning, pain and quality of life after amputation for musculoskeletal tumours: a national survey. Bone Joint J 2015;97-B:128490.

91. Hanlon M, Krajbich JI. Rotationplasty in skeletally immature patients: long-term followup results. Clin Orthop Relat Res 1999;(358):75-82.

92. Mayerson JL. Living with rotationplasty: quality of life in rotationplasty patients from childhood to adulthood. J Surg Oncol 2012;105:743-4.

93. Rodl RW, Pohlmann U, Gosheger G, et al. Rotationplasty: quality of life after 10 years in 22 patients. Acta Orthop Scand 2002;73:85-8.

94. Veenstra KM, Sprangers MA, van der Eyken JW, et al. Quality of life in survivors with a Van Ness-Borggreve rotationplasty after bone tumour resection. J Surg Oncol 2000;73: 192-7.

95. Ackman J, Altiok H, Flanagan A, et al. Long-term follow-up of Van Nes rotationplasty in patients with congenital proximal focal femoral deficiency. Bone Joint J 2013;95-B:192-8. 